\title{
Molecular characterization and upregulation of cytosolic manganese superoxide dismutase by imidazole derivative KK-42 in Macrobrachium nipponense
}

\author{
W.F. Wang ${ }^{1}$, L.M. Mu ${ }^{1}$, X.S. Wu ${ }^{2}$, H. Yang ${ }^{3}$ and Q.J. Ning ${ }^{3}$ \\ ${ }^{1}$ Department of Life Sciences and Technology, Xinxiang Medical University, \\ Xinxiang, Henan, China \\ ${ }^{2}$ Department of Vasculocardiology, The 371 Central Hospital of PLA, \\ Xinxiang, Henan, China \\ ${ }^{3}$ College of Life Sciences, Henan Normal University, Xinxiang, Henan, China \\ Corresponding author: Q.J. Ning \\ E-mail:wwf200594@163.com \\ Genet. Mol. Res. 15 (3): gmr.15038484 \\ Received January 25, 2016 \\ Accepted April 15, 2016 \\ Published August 29, 2016 \\ DOI http://dx.doi.org/10.4238/gmr.15038484
}

Copyright (C) 2016 The Authors. This is an open-access article distributed under the terms of the Creative Commons Attribution ShareAlike (CC BY-SA) 4.0 License.

\begin{abstract}
Imidazole derivative KK-42 is a well-known regulator of insect growth. KK-42 pretreatment has been shown to promote the survival of Macrobrachium nipponense infected with Aeromonas hydrophila, possibly via activation of superoxide dismutase (SOD). In this study, the cytMnSOD gene was cloned from the hepatopancreas of $M$. nipponense using the rapid amplification of cDNA ends technique. The full-length cDNA of $c y t M n S O D$ was 1233 bp long, and the open reading frame was 858 bp long, encoding a 286 -aa protein with a 60 aa leader sequence. The calculated molecular mass of the translated cytMnSOD protein was $31.33 \mathrm{kDa}$, with an estimated isoelectric
\end{abstract}


point of 5.62. cytMnSOD contained two N-glycosylation sites, four conserved amino acids responsible for binding manganese, and a manganese SOD domain (DVWEHAYY). Real-time RT-PCR analysis showed that cytMnSOD was expressed in all tissues examined with the highest expression observed in the hepatopancreas. Levels of the cytMnSOD transcript in the hepatopancreas were highest in stage $\mathrm{C}$ of the molting cycle. Real-time PCR analysis revealed that cytMnSOD expression increased significantly 3,6 , and $12 \mathrm{~h}$ after KK-42 treatment, with simultaneous increases in SOD activity from 6 to $12 \mathrm{~h}$. Our results demonstrate that cytMnSOD expression and SOD activity may be induced by KK-42, which may represent one of the molecular mechanisms through which KK-42 promotes increased survival of prawns infected with $A$. hydrophila.

Key words: Macrobrachium nipponense; Expression analysis; Cytosolic manganese superoxide dismutase; KK-42

\section{INTRODUCTION}

Crustaceans lack an adaptive immune system and depend on their innate immune system to generate immune responses (Chang et al., 2013; Lin et al., 2015). The innate immune system involves non-specific molecules such as phenoloxidase, hemocyanin, lysozyme, lectins. and superoxide dismutase (SOD) (Lin et al., 2015). Previous studies have shown that immune responses, such as respiratory bursts, are created when the bodies of crustaceans are stimulated by external pathogens (Duan et al., 2015a,b). These responses can cause the production of multiple reactive oxygen species (ROS). The non-specific action of ROS means that they kill the invading pathogens but also cause serious damage to the organs, tissues, and cells of the host (Sun et al., 2014a). This leads to physiological and immune dysfunction. Antioxidant enzymes, such as SOD, are used to eliminate excessive ROS in individuals infected with pathogens in order to maintain normal cellular metabolism. Determination of SOD gene expression and enzymatic activity are commonly used to measure the levels of immunity within crustaceans (Duan et al., 2015a). The SOD enzymes, which include cytosolic manganese SOD (cytMnSOD) and mitochondrial manganese SOD (mtMnSOD), are widespread and exist in almost all aerobic organisms (Xie et al., 2012; Zhao et al., 2014). The SOD genes have been cloned from crustaceans such as Macrobrachium rosenbergii (Cheng et al., 2006), Fenneropenaeus chinensis (Zhang et al., 2007), and Marsupenaeus japonicus (Lin et al., 2010). However, the Macrobrachium nipponense cytMnSOD gene has not yet been cloned.

KK-42 is an imidazole derivative, which is used as a growth regulator in insects and has not been used frequently in crustaceans (Berghiche et al., 2005; Liu et al., 2015). Findings from our previous studies revealed that KK-42 can promote the larval growth of Litopenaeus vannamei and improve the survival rate (Ning et al., 2007). The results of recent studies have also shown that KK-42 can enhance the survival of M. nipponense when challenged with the Aeromonas hydrophila bacterium (Wang et al., 2013). To investigate the possible mechanism through which KK-42 promotes survival, we have cloned the M. nipponense cytMnSOD gene, measured its temporal-spatial expression, and investigated SOD enzyme activity before and after KK-42 treatment.

Genetics and Molecular Research 15 (3): gmr.15038484 


\section{MATERIAL AND METHODS}

\section{Prawns}

Healthy prawns with a body length of $4.5 \pm 0.5 \mathrm{~cm}$ in the intermolt stage were obtained from a commercial fishing farm near Yuanyang, Henan Province, China, and acclimatized at $27^{\circ} \pm 1^{\circ} \mathrm{C}$ in running-water tanks in the laboratory for 2 weeks prior to experimentation (Wang et al., 2015). Two groups of 120 prawns were pretreated with KK-42 (purity $\geq 95 \%$, Department of Chemistry, Yantai University, China) as previously described, at a concentration of 0 (control) or $1.95 \times 10^{-4} \mathrm{M}$ (treatment) (Wang et al., 2013). At 3, 6, 12, 24, and $48 \mathrm{~h}$ after KK42 treatment, individual hepatopancreas were collected to examine cytMnSOD expressions and SOD activity. All experiments were performed in triplicate.

\section{Total RNA isolation and reverse transcription (RT)}

Total RNA was extracted using TRIzol (Invitrogen Life Technologies, USA) according to the manufacturer protocol. Total RNA $(5 \mu \mathrm{g})$ was reverse transcribed using M-MLV FirstStrand cDNA synthesis Kit (TaKaRa, Japan), according to the manufacturer instructions.

\section{cytMnSOD cDNA cloning and sequencing}

The full-length cytMnSOD of M. nipponense was obtained with rapid amplification of cDNA ends (RACE) methodology using hepatopancreas cDNA as the template. To obtain partial cDNA sequences, two degenerate primers, P1 (5'-gttYKccYatatcaatgctg-3') and P2 (5'-cKRaggttcttgtactgaMgg-3'), were designed based on the highly conserved nucleotides of known cytMnSOD of arthropods. According to the above cDNA sequences, specific primers (5'-Race outer primer-5'-ATGTGTAAGCTGAAGGGGGTCC-3', 5'-Race inner primer-5'CAAGCCAGCCCCAACCAGAA-3'; 3'-Race outer primer-5'-TCACGTTCTTCCTCCCCTG A-3', 3'-Race inner primer-5'-TTCTGGTTGGGGCTGGCTTG-3') were designed to characterize the 5'- and 3'-regions of the cytMnSOD cDNA by RACE-PCR (TaKaRa) according to the manufacturer protocol. The full-length PCR product was cloned into pMDT19 (TaKaRa) and sequenced from both directions by a commercial sequencing company (Invitrogen). Finally, the full-length SOD cDNA fragment of M. nipponense was obtained by overlapping three cDNA fragment sequences.

Sequences were compared and analyzed with the BLAST algorithm at the National Center for Biotechnology Information (NCBI). Potential N-glycosylation sites and signal peptide were predicted by NetNGlyc 1.0 Server (http://www.cbs.dtu. dk/services/NetNGlyc/) and SignalP program, respectively.

\section{Temporal and spatial expression of cytMnSOD by real-time PCR}

RNA was isolated from hematocytes as previously described (Wang et al., 2013). The other tissues (hepatopancreas, muscle, gill, intestine, mandibular organ, and ovary) were dissected and snap-frozen in liquid nitrogen for RNA isolation and cDNA synthesis. The molt cycle is divided into several stages, including postmolt (A, B), intermolt (C), and premolt (D0, D1, D2, and D3) (Cheng et al., 2006). Five molt stages (A, B, C, D0-1, and D2-3) were used

Genetics and Molecular Research 15 (3): gmr.15038484 
to examine cytMnSOD expression in the hepatopancreas (Wang et al., 2015).

mRNA expression of cytMnSOD in various tissues and different molt stages was analyzed by quantitative real-time PCR using SYBR Premix Ex Taq ${ }^{\mathrm{TM}}$ (TaKaRa) following the manufacturer instructions. Gene-specific primers P-F (5'-CCCAAATGGAGGTGGAGAAC$3^{\prime}$ ) and P-R (5'-TAGGACAGTAGCCAAGCCAGC-3'), as well as primers for the internal standard 18S rRNA, 18SRNA-F (5'-TGTTACGGGTGACGGAGAA-3') and 18SRNA-R (5'-CATTCCAATTACGCAGACTCGG-3'), were designed based on the sequences of prawn M. nipponense (GenBank accession Nos. HQ852226.1 and DQ531769.1). PCR was performed using an ABI 7500 Real-Time Detection System (Applied Biosystems, USA) under the following conditions: initial denaturation at $95^{\circ} \mathrm{C}$ for $30 \mathrm{~s}$ and amplification for 40 cycles $\left(95^{\circ} \mathrm{C}, 5 \mathrm{~s} ; 60^{\circ} \mathrm{C}\right.$, $34 \mathrm{~s})$. Amplifications were carried out on a 96 -well plate in a $20-\mu \mathrm{L}$ reaction volume containing $10 \mu \mathrm{L}$ SYBR Premix Ex Taq ${ }^{\mathrm{TM}}, 0.4 \mu \mathrm{L}$ ROX reference dye II, $0.4 \mu \mathrm{L}$ each of forward and reverse primers, $2 \mu \mathrm{L}$ diluted cDNA, and sterile-water to adjust the reaction volume (Wang et al., 2013). The real-time quantitative PCR data were analyzed by the $2^{-\Delta \Delta \mathrm{Ct}}$ method.

\section{Measuring SOD activity}

SOD activity was measured using an SOD kit (Nanjing Jiancheng Bioengineering Institute, China). Briefly, $30 \mathrm{~mL}$ hemolymph supernatant $(50 \mathrm{mg} / \mathrm{mL})$ or $50 \mathrm{~mL}$ hepatopancreas supernatant $(5 \mathrm{mg} / \mathrm{mL})$ were used. SOD activity is reported as $\mathrm{U} / \mathrm{mL}$ for hemolymph or $\mathrm{U} / \mathrm{mg}$ for hepatopancreas. The assay was carried out in triplicate.

\section{Statistical analysis}

All data are reported as means $\pm \mathrm{SE}$ and were derived from three independent experiments. A multiple-comparison Duncan test was used to compare significant differences in cytMnSOD gene expression and SOD activity among groups using the SPSS13.0 software. A significance level of $\mathrm{P}=0.05$ was selected.

\section{RESULTS}

\section{Cloning and characterization of the cytMnSOD gene}

The $c y t M n S O D$ sequence and the deduced amino acid sequence are shown in Figure 1. The full-length cDNA of $c y t M n S O D$ was 1233 bp long containing a 73-bp 5'-UTR, a 302bp 3'-UTR with a stop codon, a polyadenylation site (AATATA), and a poly(A) tail. The open reading frame was $858 \mathrm{bp}$, encoding a protein of 286 aa. The calculated molecular mass of the mature protein was $31.33 \mathrm{kDa}$, with an estimated isoelectric point of 5.62. The cytMnSOD sequence and its deduced amino acid sequence have been submitted to NCBI GenBank under accession No. HQ852226.1.

The deduced amino acid sequence contains a 60 -aa leader peptide sequence in the $\mathrm{N}$-terminus, and two N-glycosylation sites (NHT and NMS), four conserved amino acids that bind manganese (H110, H158, D243, and H247), and a manganese SOD domain (DVWEHAYY) (Figure 1). The deduced amino acid sequence of $M$. nipponense cytMnSOD was $98,81,79$, and $78 \%$ similar to that of cytMnSOD from M. rosenbergii, Procambarus clarkii, Litopenaeus vannamei, and M. japonicus, respectively.

Genetics and Molecular Research 15 (3): gmr.15038484 


\begin{tabular}{|c|c|}
\hline & \\
\hline & AAGTATCCCGAACATTCCCTCGCCAACCGCCACCTTTTGTCTGAAAGACCACGATCAAGC \\
\hline & ATGGCTGACAAGGATGCATACATCAGTGCCCTGGAGCAGAAGTTC \\
\hline & $\begin{array}{llllllllllllllllllll}M & A & D & K & D & A & Y & I & S & A & L & E & Q & K & L & A & E & L & S & G \\
\end{array}$ \\
\hline & ATTGAAGTAGATCAAATAAAAAAGAATCAGCTCGCAAATGCTGCCGACGAGGCCCGTGTC \\
\hline & $\begin{array}{llllllllllllllllllll}\mathrm{I} & \mathrm{E} & \mathrm{V} & \mathrm{D} & \mathrm{Q} & \mathrm{I} & \mathrm{K} & \mathrm{K} & \mathrm{N} & \mathrm{Q} & \mathrm{L} & \mathrm{A} & \mathrm{N} & \mathrm{A} & \mathrm{A} & \mathrm{D} & \mathrm{E} & \mathrm{A} & \mathrm{R} & \mathrm{V} \\
\end{array}$ \\
\hline & ATTGGCGAAATGGCCGCTTACGTTGCCAGCATCACTGTTCAGCGAGCCGCCGATGCCCA \\
\hline & $\begin{array}{lllllllllllllllllll}I & G & E & M & A & A & Y & V & A & S & I & T & V & Q & R & A & A & D & A \\
\end{array}$ \\
\hline & GTAGGACTTGTCAGCCCCCAGATTGCCGAAATTTATTCCCATATCAATGCTGAACTGAGT \\
\hline & $\begin{array}{llllllllllllllllllll}V & G & L & V & S & P & Q & I & A & E & I & Y & S & H & I & N & A & E & L & S\end{array}$ \\
\hline & GAGGCCCGTGGTGCTCACGTTCTTCCTCCCCTGAAATACGACTACAATGCATTGGAGCCT \\
\hline & 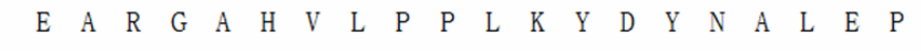 \\
\hline & CACATTTCTGGTACCATCATGGAAATCCATCACACCAAACATCAC \\
\hline & $\begin{array}{llllllllllllllllllll}H & I & S & G & T & I & M & E & I & H & H & T & K & H & H & Q & A & Y & I & N\end{array}$ \\
\hline & AACCTTAAAGCTGCAACGGATAAGTTGATAGATGCTGAACAGAACAATGATGTAAATGCC \\
\hline & $\begin{array}{llllllllllllllllllll}N & L & K & A & A & T & D & K & L & I & D & A & E & Q & N & N & D & V & N & A\end{array}$ \\
\hline & ATGAATGCACTGTTACCTGCCATCAAGTTCAACGGAGGTGGTCACCTGAACCACACCATC \\
\hline & 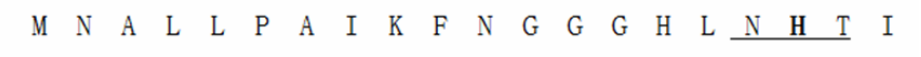 \\
\hline & TTCTGGACCAACATGAGCCCAAATGGAGGTGGAGAACCAAGTGGATCAGTTGCTGATGCC \\
\hline & $\begin{array}{llllllllllllllllllll}F & W & T & N & M & S & P & N & G & G & G & E & P & S & G & S & V & A & D & A\end{array}$ \\
\hline & ATTAATGCTGAATTTGGCTCATTTCAGGCATTTAAGGATAAGTTTTCTGCCGCTAGTGTG \\
\hline & $\begin{array}{llllllllllllllllllll}I & N & A & E & F & G & S & F & Q & A & F & K & D & K & F & S & A & A & S & V\end{array}$ \\
\hline & GGTGTGAAAGGTTCTGGTTGGGGCTGGCTTGGCTACTGTCCTAAAAACGACAAAGTTGCC \\
\hline & 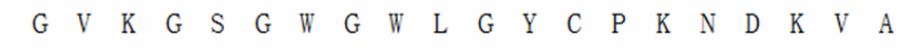 \\
\hline & GTTGCCACTTGCCAAAACCAGGACCCCCTTCAGCTTACACATGGATTGGTACCACTCCTT \\
\hline & 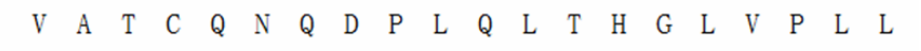 \\
\hline & GGACTGGATGTATGGGAACACGCTTACTATCTACAGTACAAGAACCTGAGGGCTGACTAC \\
\hline & $\begin{array}{lllllllllllllllllllll}G & L & D & V & \text { W } & \text { E } & \text { H } & \text { A } & \text { Y } & \text { Y } & \text { L } & \text { Q } & \text { Y } & \text { K } & \text { N } & \text { L } & \text { R } & \text { A } & \text { D } & \text { Y }\end{array}$ \\
\hline & GTCAAAGCTTTCTTCAATGTGATCGACTGGTCTAACGTAAACGACCGCTACGAAAATGCC \\
\hline & $\begin{array}{llllllllllllllllllll}V & K & A & F & F & N & V & I & D & W & S & N & V & N & D & R & Y & E & N & A\end{array}$ \\
\hline & CGCAAGGCCGCTGGACACTGACTTCTCTATACTGATCAATACCTTATAACTAACAAAGGC \\
\hline & A $A$ A $\quad H \quad *$ \\
\hline & A \\
\hline & ITT \\
\hline & TTTTG \\
\hline & TCTC \\
\hline & \\
\hline
\end{tabular}

Figure 1. Nucleotide sequence of the cDNA encoding cytMnSOD from Macrobrachium nipponense hepatopancreas and its deduced amino acid sequence. Potential N-glycosylation sites are underlined. The putative cytMnSOD signature is shown in white letters against a black background. Putative manganese binding sites are in bold (H110, H158, D243, and H247). The leader sequence peptide of cytMnSOD is underlined twice. 


\section{Temporal and spatial expression of cytMnSOD}

Real-time PCR was employed to measure the distribution of $c y t M n S O D$ mRNA in different tissues. The mRNA of cytMnSOD was detected in muscle, hepatopancreas, ovary, hemocytes, gill, and mandibular organ tissues, with the highest and lowest expression observed in the hepatopancreas and mandibular organ, respectively (Figure 2).

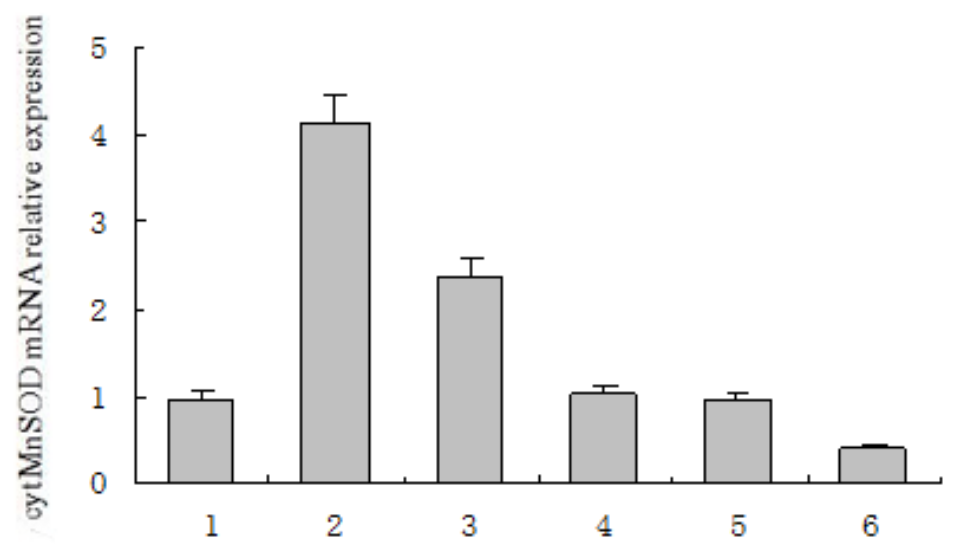

Figure 2. Real-time PCR analysis of cytMnSOD transcripts from different tissues. 1: muscle, 2: hepatopancreas, 3 : ovary, 4: hemocytes, 5: gill, and 6: mandibular organ.

Considering that $c y t M n S O D$ mRNA expression was high in the hepatopancreas, we selected this tissue to measure mRNA expression in prawns at different molt stages. The results indicated that fluctuations in the transcript level of $c y t M n S O D$ in the hepatopancreas were affected by the molt cycle. cytMnSOD mRNA expression was detected throughout the entire molt cycle, with the highest expression observed in stage $C$, and no significant differences were observed between stages A, B, D0-1, and D2-3 (Figure 3).

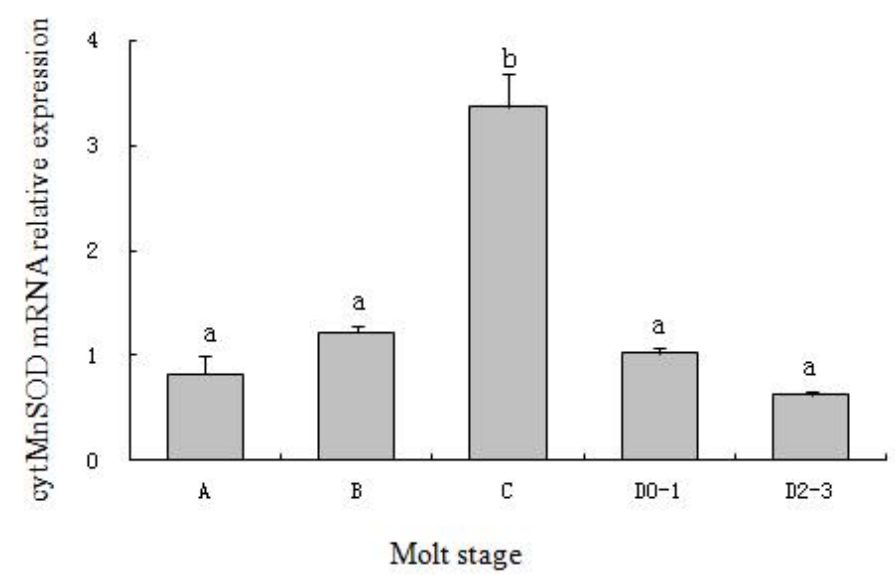

Figure 3. Relative expression of $c y t M n S O D$ in the hepatopancreas during the molt cycle. Different letters indicated a significant difference $(\mathrm{P}<0.05)$.

Genetics and Molecular Research 15 (3): gmr.15038484 


\section{Induction of cytMnSOD expression and SOD activity in response to KK-42}

KK-42 treatment was found to induce cytMnSOD transcription in the hepatopancreas. At $3 \mathrm{~h}$ after treatment, the expression of cytMnSOD mRNA was significantly upregulated, with the highest level observed $6 \mathrm{~h}$ after treatment. Expression was downregulated at $12 \mathrm{~h}$. At 3, 6, and $12 \mathrm{~h}$ after treatment, the mRNA levels were markedly increased relative to those of the control $(\mathrm{P}<0.05)$; however, cytMnSOD expression in the control group did not change significantly during the experiment (Figure 4).

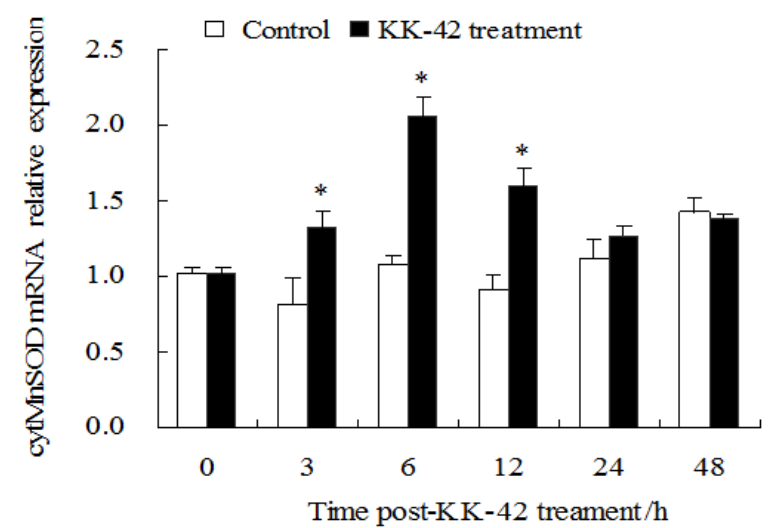

Figure 4. cytMnSOD mRNA levels in the prawn hepatopancreas following KK-42 treatment. Bars represent means \pm SE. $* \mathrm{P}<0.05$ vs the control group.

SOD activity in the hepatopancreas of prawns in the control group varied, but no statistically significant differences were observed compared with the activity at $0 \mathrm{~h}$. SOD activity increased significantly from 6 to $12 \mathrm{~h}$ in KK-42-treated prawns, peaking at $12 \mathrm{~h}$ (Figure 5) compared with the activity in the corresponding control group $(\mathrm{P}<0.05)$, demonstrating that SOD activity, as well as cytMnSOD mRNA expression, could be induced by KK-42.

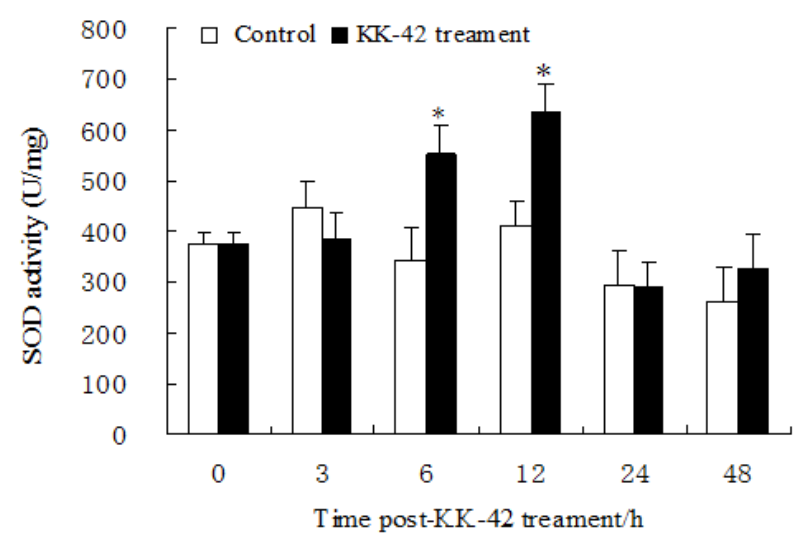

Figure 5. Superoxide dismutase (SOD) activity levels in the hepatopancreas of prawn following KK-42 treatment. Bars represent means $\pm \mathrm{SE} . * \mathrm{P}<0.05$ vs the control group. 


\section{DISCUSSION}

We have successfully cloned the M. nipponense cytMnSOD gene and determined that the amino acid sequence contains an MnSOD characteristic peptide (DVWEHAYY) and four manganese ion binding sites. The cytMnSOD and mtMnSOD enzymes are encoded by different genes, and the most clear differences were found within the N-terminus of the proteins (Lin et al., 2010). When compared with mtMnSOD, cytMnSOD contains an additional extended region of 60 aa at the $\mathrm{N}$-terminus. The 60 th aa is a putative proteolytic cleavage site (Gabbianelli et al., 1997). The predicted molecular mass of the mature peptide was $24.8 \mathrm{kDa}$, which is similar to that found for mature peptides from M. rosenbergii and Callinectes sapidus (Brouwer et al., 2003; Cheng et al., 2006).

Crustacean molting is a complex physiological process, which may be influenced by physiological states and environmental signals (Engel et al., 2001; Kuballa et al., 2011). Few studies have investigated the relationship between the molting cycle and MnSOD expression. Previous studies revealed that cytMnSOD activity was highest in the hepatopancreas of $C$. sapidus at intermolt, with little activity detected at postecdysis. However, mtMnSOD activity was successfully detected during the molting cycle (Brouwer et al., 2003). Our results revealed that fluctuations in the expression of cytMnSOD were influenced by the molting cycle, and that the highest level of expression was observed during intermolt, suggesting that transcription of this gene may be regulated by ecdysone. Moreover, marked changes in oxygen consumption during the molting cycle may affect MnSOD expression (Engel et al., 2001; Schvezov et al., 2015).

Our results showed that cytMnSOD was expressed in all the examined tissues, which is consistent with the results of studies in other animals (Wang et al., 2007; Sun et al., 2014b) The widespread expression of MnSOD is related to its function. The hepatopancreas and hemocytes are the main organs of the immune system, and the gills are involved in filtration and pathogen removal. We hypothesize that cytMnSOD is an important antioxidant, which may help to maintain the normal function of tissues.

In addition to its effect as a growth regulator in insects, we identified a further novel role for KK-42. Pretreatment with KK-42 can increase the survival of M. nipponense following challenge with $A$. hydrophila; however, the mechanism responsible is unclear. Due to the high levels of $M n S O D$ expression at intermolt in the hepatopancreas, we selected intermolt individuals as experimental samples. The hepatopancreas is an important organ in crustaceans and has many physiological functions, including digestion, absorption, and metabolism. It is very sensitive to environmental changes and is involved in protective immune responses (Sun et al., 2014b). The results of the present study indicated that KK-42 treatment can induce cytMnSOD gene expression, as observed $3 \mathrm{~h}$ after treatment. Peak expression was observed $6 \mathrm{~h}$ after treatment, and changes in the enzymatic activity of SOD were generally consistent with changes in gene expression. Previous studies have also demonstrated that SOD activity increases in hemocytes and muscle cells of $L$. vannamei treated with an immunopotentiator, and the survival rate increased following bacterial infection, suggesting that SOD regulates the immune response in shrimps (Campa-Cordova et al., 2002). In crustaceans, SOD and phenoloxidase are two enzymes with roles in the immune system, which may be used as indicator enzymes to detect immune function (Amparyup et al., 2013; Liu et al., 2013). Induction of cytMnSOD gene expression by KK-42 is advantageous for adaptation to harsh environments as it strengthens immunity against pathogenic bacteria and viruses and enhances tolerance, which is likely to be one of the molecular mechanisms through which KK-42 increases survival of prawns infected with A. hydrophila.

Genetics and Molecular Research 15 (3): gmr.15038484 
In conclusion, the cytMnSOD gene was first cloned from the hepatopancreas of $M$. nipponense. The present results showed that KK-42 administration can activate immune defense in crustaceans against highly pathogenic bacterium by increasing cytMnSOD gene expression and SOD activity. This is suggested to contribute to the increase in $M$. nipponense survival rate; however, the precise regulatory pathway through which KK-42 exerts its effects remains to be determined.

\section{Conflicts of interest}

The authors declare no conflict of interest.

\section{ACKNOWLEDGMENTS}

Research supported by grants from the Henan Scientific and Technological Research Projects (\#142102310045), the Natural Science Project of the Education Department of Henan Province (\#14A240003), and the Cultivate Foundation of Xinxiang Medical College (\#2013ZD109).

\section{REFERENCES}

Amparyup P, Charoensapsri W and Tassanakajon A (2013). Prophenoloxidase system and its role in shrimp immune responses against major pathogens. Fish Shellfish Immunol. 34: 990-1001.http://dx.doi.org/10.1016/j.fsi.2012.08.019

Berghiche H, Houamria M, Smagghe G and Soltani N (2005). Activity of kk-42 in combinated treatment with RH-0345 or 20-hydroxyecdysone on morphometric measurements and free ecdysteroid in eggs of mealworms. Commun. Agric. Appl. Biol. Sci. 70: 837-841.

Brouwer M, Hoexum Brouwer T, Grater W and Brown-Peterson N (2003). Replacement of a cytosolic copper/ zinc superoxide dismutase by a novel cytosolic manganese superoxide dismutase in crustaceans that use copper (haemocyanin) for oxygen transport. Biochem J. 374: 219-228. http://dx.doi.org/10.1042/bj20030272

Campa-Cordova AI, Hernandez-Saavedra NY and Ascencio F (2002). Superoxide dismutase as modulator of immune function in American white shrimp (Litopenaeus vannamei). Comp. Biochem. Physiol. C. Toxicol. Pharmacol. 133: 557-565. http://dx.doi.org/10.1016/S1532-0456(02)00125-4

Chang CC, Rahmawaty A and Chang ZW (2013). Molecular and immunological responses of the giant freshwater prawn, Macrobrachium rosenbergii, to the organophosphorus insecticide, trichlorfon. Aquat. Toxicol. 131: 18-26. http:// dx.doi.org/10.1016/j.aquatox.2012.12.024

Cheng W, Tung YH, Liu CH and Chen JC (2006). Molecular cloning and characterisation of cytosolic manganese superoxide dismutase (cytMn-SOD) from the giant freshwater prawn Macrobrachium rosenbergii. Fish Shellfish Immunol. 20: 438-449. http://dx.doi.org/10.1016/j.fsi.2005.05.016

Duan Y, Zhang J, Dong H, Wang Y, et al. (2015a). Effect of desiccation and resubmersion on the oxidative stress response of the kuruma shrimp Marsupenaeus japonicus. Fish Shellfish Immunol. 14: 91-99.

Duan Y, Zhang J, Dong H, Wang Y, et al. (2015b). Oxidative stress response of the black tiger shrimp Penaeus monodon to Vibrio parahaemolyticus challenge. Fish Shellfish Immunol. 46: 354-365.http://dx.doi.org/10.1016/j.fsi.2015.06.032

Engel DW, Brouwer M and Mercaldo-Allen R (2001). Effects of molting and environmental factors on trace metal bodyburdens and hemocyanin concentrations in the American lobster, Homarus americanus. Mar. Environ. Res. 52: 257269. http://dx.doi.org/10.1016/S0141-1136(01)00098-8

Gabbianelli R, Battistoni A, Polticelli F, Meier B, et al. (1997). Effect of Lys175 mutation on structure function properties of Propionibacterium shermanii superoxide dismutase. Protein Eng. 10: 1067-1070. http://dx.doi.org/10.1093/ protein/10.9.1067

Kuballa AV, Holton TA, Paterson B and Elizur A (2011). Moult cycle specific differential gene expression profiling of the crab Portunus pelagicus. BMC Genomics 12: 1471-2164.http://dx.doi.org/10.1186/1471-2164-12-147

Lin YC, Lee FF, Wu CL and Chen JC (2010). Molecular cloning and characterization of a cytosolic manganese superoxide dismutase (cytMnSOD) and mitochondrial manganese superoxide dismutase (mtMnSOD) from the kuruma shrimp Marsupenaeus japonicus. Fish Shellfish Immunol. 28: 143-150.http://dx.doi.org/10.1016/j.fsi.2009.10.012

Genetics and Molecular Research 15 (3): gmr.15038484 
Lin YC, Chen JC, Chen YY, Yeh ST, et al. (2015). Crowding of white shrimp Litopenaeus vananmei depresses their immunity to and resistance against Vibrio alginolyticus and white spot syndrome virus. Fish Shellfish Immunol. 45: 104-111.http://dx.doi.org/10.1016/j.fsi.2015.02.012

Liu Y, Zhang Q and Denlinger DL (2015). Imidazole derivative KK-42 boosts pupal diapause incidence and delays diapause termination in several insect species. J. Insect Physiol. 74: 38-44.http://dx.doi.org/10.1016/j.jinsphys.2015.02.003

Liu YT, Chang CI, Hseu JR, Liu KF, et al. (2013). Immune responses of prophenoloxidase and cytosolic manganese superoxide dismutase in the freshwater crayfish Cherax quadricarinatus against a virus and bacterium. Mol. Immunol. 56: 72-80. http://dx.doi.org/10.1016/j.molimm.2013.03.023

Ning QJ, Fu SG, Xu XJ and He JT (2007). A new and practical application of JH antagonist KK-42 to promoting growth of shrimp Penaeus schmitti. Aquaculture 270: 422-426. http://dx.doi.org/10.1016/j.aquaculture.2007.04.078

Schvezov N, Lovrich GA, Florentin O and Romero MC (2015). Baseline defense system of commercial male king crab Lithodes santolla from the Beagle Channel. Comp. Biochem. Physiol. A Mol. Integr. Physiol. 181: 18-26. http:// dx.doi.org/10.1016/j.cbpa.2014.11.016

Sun H, Xu XY, Tian XL, Shao HT, et al. (2014a). Activation of NF-kappaB and respiratory burst following Aspergillus fumigatus stimulation of macrophages. Immunobiology 219: 25-36. http://dx.doi.org/10.1016/j.imbio.2013.06.013

Sun S, Zhu J, Jiang X, Li B, et al. (2014b). Molecular cloning, tissue distribution and expression analysis of a manganese superoxide dismutase in blunt snout bream Megalobrama amblycephala. Fish Shellfish Immunol. 38: 340-347. http:// dx.doi.org/10.1016/j.fsi.2014.03.036

Wang WF, Yang H, Liu F, Chen XL, et al. (2013). A novel effect of imidazole derivative KK-42 on increasing survival of Aeromonas hydrophila challenged prawn Macrobrachium nipponense. Fish Shellfish Immunol. 34: 167-172. http:// dx.doi.org/10.1016/j.fsi.2012.10.027

Wang WF, Mu LM, Wu XS, Yang H, et al. (2015). Molecular cloning, characterization and expression analysis of mitochondrial manganese superoxide dismutase of an oriental river prawn, Macrobrachium nipponense. Pakistan J. Zool. 47: 933-942.

Wang YC, Chang PS and Chen HY (2007). Tissue expressions of nine genes important to immune defence of the Pacific white shrimp Litopenaeus vannamei. Fish Shellfish Immunol. 23: 1161-1177. http://dx.doi.org/10.1016/j. $\underline{\text { fsi.2007.04.004 }}$

Xie XQ, Li F, Ying SH and Feng MG (2012). Additive contributions of two manganese-cored superoxide dismutases (MnSODs) to antioxidation, UV tolerance and virulence of Beauveria bassiana. PLoS One 7: e30298. http://dx.doi. org/10.1371/journal.pone.0030298

Zhang Q, Li F, Wang B, Zhang J, et al. (2007). The mitochondrial manganese superoxide dismutase gene in Chinese shrimp Fenneropenaeus chinensis: cloning, distribution and expression. Dev. Comp. Immunol. 31: 429-440. http:// dx.doi.org/10.1016/j.dci.2006.08.005

Zhao DX, Chen LQ, Qin JG, Qin CJ, et al. (2014). Molecular characterization of a cytosolic manganese superoxide dismutase from the Chinese mitten crab, Eriocheir sinensis. Genet. Mol. Res. 13: 9429-9442. http://dx.doi. org/10.4238/2014.November.11.8

Genetics and Molecular Research 15 (3): gmr.15038484 